don't. In the trochlear nucleus of a bird, just as in the ventral horn of its spinal cord, half the motoneurones die (Cowan in Development and Aging of the Nervous System (ed. Rockstein), Academic Press, 1973).

The evidence so far has not yielded a solution to the puzzle of motoneurone death; but it has at least proved that the solution will be interesting.

\section{Birds see ultraviolet light}

from J.K. Bowmaker

HUMAN colour vision extends from about $400 \mathrm{~nm}$ in the violet to about $750 \mathrm{~nm}$ in the red. Why are we restricted to this limited range? About $80 \%$ of the electromagnetic radiation reaching the Earth's surface lies between $300 \mathrm{~nm}$ in the ultraviolet and 1,100 $\mathrm{nm}$ in the infrared, but nevertheless only part of this spectrum is available for vision since radiation with wavelengths longer than about $800 \mathrm{~nm}$ has too little energy to cause photochemical reactions. Thus there is available for vision a spectral range from about $300 \mathrm{~nm}$ to $800 \mathrm{~nm}$. However, the limits of an animal's vision are determined by the visual pigments contained within the photopic receptors of the retina, the cones, and by pre-receptoral filters. In man these are principally the lens that cuts off light below about $400 \mathrm{~nm}$, and the macula pigment in the yellow spot of the retina. Filtering violet and near ultraviolet light reduces chromatic aberration which becomes increasingly severe at short wavelengths.

The question remains whether other species are similarly restricted. It is natural perhaps to compare the visual sense of other diurnal species with our own, on the assumption that we represent the culmination of the evolution of colour vision. But is this reasonable? The early mammals, around at the time of the dinosaurs, were probably nocturnal, resulting in their colour vision becoming largely degenerate, with many modern mammals having only limited dichromatic vision. The Old World primates, including man, are somewhat of an exception in that they have evolved secondarily trichromatic vision along with the adoption of diurnal habits. Even so our trichromacy has a 'blue' channel that is about 100 times less sensitive than the 'red' and 'green' channels. The true culmination of the evolution of colour vision in vertebrates would be expected to be found in highly evolved diurnal animals, perhaps best represented by diurnal birds and it is

J.K. Bowmaker is a Lecturer in the Department of Zoology and Comparative Physiology, Queen Mary College, London within these species that we should look for colour vision making use of more of the available spectrum.

The most striking feature of the retina of diurnal birds is the presence of brightly coloured oil droplets contained within the cones that act as highly selective long-pass cut-off filters (see Bowmaker Vision Res. $17 ; 1129 ; 1979)$. The lenses of most diurnal birds are optically clear and it seems that one of the functions of the oil droplets is to limit chromatic aberration selectively at the receptor level. However, amongst the coloured oil droplets there are colourless ones that presumably transmit near ultraviolet light with the possibility that diurnal birds can detect these short wavelengths as a 'colour', different in hue from the rest of their visible spectrum.

It is known that pigeons can see in this spectral region (Wright $J$. exp. anal. Behav. 17, $325 ; 1972$ ) and in a recent paper Timothy Goldsmith (Science 207, 786; 1980) has presented evidence that several species of humming bird can discriminate the near ultraviolet. Working with a natural population of the birds he set them a simple task of selecting artificial feeders containing a sugar solution on the basis of a coloured viewing screen set in the feeders. The birds were able to distinguish the near ultraviolet around $370 \mathrm{~nm}$ from darkness or from the small amount of far red light that leaked through the ultraviolet transmitting glass filter, which human observers were unable to do. The birds could also distinguish white light lacking wavelengths below 400 $\mathrm{nm}$ from white light from a quartz-halogen bulb that does contain near ultraviolet radiation.

The near ultraviolet sensitivity of the humming birds presumably involves cones containing an oil droplet that transmits short wavelengths but also a visual pigment that is sensitive to these wavelengths. Evidence from pigeons and chickens (Graf \& Norren Vision Res. 14; 1203; 1974) indicates that in addition to red, green and blue-sensitive visual pigments there is a visual pigment with maximum sensitivity at about $400-415 \mathrm{~nm}$. This is very close to the maximum sensitivity of the visual pigment of human 'blue' cones $(415-420 \mathrm{~nm})$ recently recorded by Bowmaker and Dartnall (J. Physiol. Lond. 298, 501; 1980 ), but the effective maximum sensitivity of the human cones is displaced to about $440 \mathrm{~nm}$ with a marked cut off below about $400 \mathrm{~nm}$ due to the filtering of the lens.

The other major group of animals known to have vision in the ultraviolet is the insects. Here chromatic aberration is not a problem since arthropod eyes are designed on different optical principles. These insects, principally bees and moths, have mouthparts designed to suck nectar from flowers, and many of the flowers rely on their insect visitors for pollination. Many such flowers reveal striking patterns of 'nectar guides' if photographed using ultraviolet sensitive film that are not visible to a human observer and it is assumed that the evolution of such guides has paralleled the evolution of ultraviolet vision in these pollinating insects. It would be interesting to know whether the flowers usually frequented by humming birds have similar ultraviolet nectar guides. However, as Goldsmith says "the interplay of several spectral classes of oil droplet with several cone pigments and the presence of receptors functioning in the near UV suggests that avian colour vision possesses a richness beyond our ken".

\section{BL Lacertae Objects the 'missing link'?}

\section{from a Correspondent}

A RECENT study of a sample of extragalactic radio sources belonging to the so-called BL Lacertae class has shed some new light on one of the most interesting questions in extragalactic radio astronomy, namely the relationship between the radio sources associated with galaxies and those associated with quasars.

Until 1968 the optical objects found to be associated with extragalactic radio sources could be divided into two basic classes, galaxies and quasars. The galaxies have a variety of forms, basically appearing diffuse on photographs; their optical spectra are also varied, ranging from the thermal continua and weak absorption lines typical of old stars to the non-thermal continua and strong emission lines which are indicative of violent activity in the galactic nucleus. This latter type of spectrum is also typical of the quasars which appear starlike on photographs and are generally at higher redshifts than the galaxies. In 1968 an apparently new class of identification was found when the radio source VRO 42.22.01 was identified with the so-called 'variable star' BL Lacertae. The radio source itself was very unusual, showing striking, rapid variations in radio flux density and in polarization. In this respect it bore similarities to the very compact sources associated with some quasars though its variations were more extreme. However, the optical spectrum of the 'star' was apparently completely featureless showing neither emission nor absorption lines, the continuum being clearly non-thermal in origin; the optical radiation was also strongly polarized and very variable in intensity. The absence of lines in the spectrum made this very peculiar object particularly tantalizing as there was no means by which to determine its redshift and its distance.

Subsequently many more sources of this type, now called BL Lac objects, have been found and to date roughly 60 are known. 